\title{
Patients with schizophrenia found that clozapine improved their wellbeing without resulting in extrapyramidal motor side effects
}

\author{
Angermeyer MC, Löffler W,Müller P, et al. Patients'and relatives'assessment of clozapine treatment. Psychol Med 2001
} Apr;31:509-17.

QUESTION: How do patients with schizophrenia experience treatment with clozapine?

\section{Design}

Semistructured, problem centred interviews.

\section{Setting}

4 psychiatric hospitals in Germany.

\section{Patients}

80 patients who were $18-60$ years of age ( $60 \%$ men), had received clozapine during inpatient or acute day hospital treatment, and were instructed to continue clozapine on an outpatient basis after discharge.

\section{Methods}

At discharge from hospital, patients were interviewed for 35-55 minutes using the "Interview on Subjective Illness Theory". An interview guide was developed especially for the interview, which included these aspects: labelling, causal attributions, perceptions of prognosis, and control attributions. Interviews were taped, transcribed, and analysed by using structured qualitative content analysis. 2 researchers independently coded the data.

\section{Main findings}

Almost one third of patients stated that they felt better after using clozapine. Patients noted improved sleeping patterns and felt calmer, more relaxed, and more balanced. They rarely mentioned the antipsychotic effect. Those who did commented that the drug caused the voices to become fainter. Some patients noted that they had brighter moods after taking clozapine and that the drug had a positive influence on their ability to work and manage their daily lives. Others felt that the drug had a protective effect-that is, they felt shielded from the negative effects of their illness. Almost one half expressed they would have a worsening of their mental state if they stopped taking the drug. Some expected they would have insomnia, restlessness, and irritability. When asked about the negative effects of the drug, patients reported fatigue and sleepiness, especially at the beginning of treatment. Some patients attributed a feeling of lack of motivation to the drug, and hypersalivation was a problem for some patients. Other reported problems were constipation, weight gain, dizziness, and cognitive deficits. Surprisingly, many patients did not know the potential risks of long term clozapine treatment. Some patients were concerned about addiction, and some anticipated that treatment with psychotropic drugs might damage inner organs, particularly the liver. Patients clearly preferred clozapine to conventional neuroleptic drugs, particularly because of the lack of extrapyramidal motor side effects. When interviewed 6 months later, a subsample of patients reported findings similar to those reported at discharge, with a greater emphasis on the fear of relapse should they stop taking the medication.

\section{Conclusions}

Patients with schizophrenia found clozapine helped them to feel better. They reported some negative effects but felt that clozapine was superior to conventional neuroleptic drugs because it stopped them having extrapyramidal motor side effects. Many patients did not, however, acknowledge the potential risks of long term treatment.

\section{COMMENTARY}

Efficacy of clozapine for treatment resistant and non-resistant schizophrenia is established, and a recent meta-analysis on al available trial based evidence confirmed the effectiveness in comparison with conventional neuroleptics. ${ }^{1}$ The authors noted, however, that global functional and pragmatic outcomes were often not reported. Approximately 30\% (range $15-45 \%$ ) of schizophrenic patients do not respond to at least 2 adequate trials of classical neuroleptics. ${ }^{2} \mathrm{~A}$ systematic review of 12 studies and meta-analysis of 7 studies for resistant schizophrenia also indicated superiority, ${ }^{3}$ although not consistently robust, over the typical antipsychotics.

This study by Angermeyer $e t a l$ is the first to report subjective evaluations of patients with schizophrenia who had been treated with several antipsychotics before starting clozapine. The authors, through the use of well designed semistructured interviews and methodology that incorporates the varied theoretical underpinnings of qualitative research, have explored the patients' experiences. The results are in keeping with those expected, with several patients reporting improvement and acceptable positive effects along with some expected negative effects. One disturbing finding was the lack of awareness of long term side effects of clozapine. The patients' relatives reported overall satisfaction with clozapine, which was noted in another study. ${ }^{4}$

It is important for clinicians to note that patients and their relatives need to be advised about the long term side effects of clozapine, and this message may have to be repeated during follow up.

Hem Raj Pal, MD

All India Institute of Medical Sciences, New Delhi, India

1 Wahlbeck K, Cheine M, Essali A, et al. Evidence of clozapine's effectiveness in schizophrenia: a systematic review and meta-analysis of randomized trials. $A m \quad J$ Psychiatry 1999;156:990-9.

2 Meltzer HY. Treatment-resistant schizophrenia-the role of clozapine. Curr Med Res Opin 1997;14:1-20.

3 Chakos M, Lieberman J, Hoffman E, et al. Effectiveness of second-generation antipsychotics in patients with treatmentresistant schizophrenia: a review and meta-analysis of randomized trials. Am J Psychiatry 2001;158:518-26.

4 Najarian SP. Family experience with positive client response to clozapine. Arch Psychiatr Nurs 1995;9:11-21.
Sources of funding:

Sandoz $A G$ and

WanderPharma

$\mathrm{GmbH}$.

For correspondence: Dr M C Angermeyer, Department of Psychiatry, University of Leipzig, Johannisallee 20, 04317 Leipzig, Germany. Fax +49 3419724509 . 